\title{
Elliptic and hyperbolic interior solutions of piecewise-constant potential vorticity geophysical vortices
}

\author{
Álvaro Viúdez $\dagger$ \\ Institut de Ciències del Mar, CSIC, 08003 Barcelona, Spain \\ (Received 27 July 2011; revised 16 January 2012; accepted 17 January 2012; \\ first published online 28 February 2012)
}

Elliptic and hyperbolic geopotential solutions, for a homogeneous distribution of potential vorticity $(\mathrm{PV})$, are obtained via $\mathrm{PV}$ inversion in geophysical vortices. The flow in the axisymmetrical three-dimensional vortices is steady and horizontal, where the centripetal acceleration plus the Coriolis acceleration equals the pressure anomaly gradient term (gradient wind or cyclo-geostrophic balance). It is found that the family of geopotential solutions in the vortex interior is completely parameterized by the PV density in the vortex and the squared aspect ratio between the horizontal and vertical semi-axes of the ellipsoidal or hyperbolic geopotential surfaces. Thus, the PV inversion task consists of obtaining, via solution of algebraic cubic equations, the absolute vertical vorticity and vertical stratification as a function of PV and aspect ratio. It is found that there is always a critical aspect ratio, which depends on PV, beyond which the PV inversion solutions are multi-valued. The complete vorticity and stratification solutions for the different regions in the PV and aspect ratio space are obtained and analysed with emphasis on the inertial and static instability of the vortex flow.

Key words: geophysical and geological flows, rotating flows, vortex dynamics

\section{Introduction}

Geophysical fluid disturbances having sharp gradients in dynamical fields, such as geophysical jets, vortices, fronts, and filaments, are associated with large potential vorticity (PV) anomalies. PV is a quantity first obtained by Beltrami in its threedimensional form (Beltrami 1871; Casey \& Naghdi 1991; Viúdez 2001), rediscovered by Rossby in the context of shallow-water dynamics and continuously stratified dynamics (Rossby 1936, 1940), and further generalized by Ertel in the case of baroclinic, diabatic and viscous flows (Ertel 1942). Under adiabatic and inviscid conditions, which are typically assumed in mid-latitude synoptic-scale motions, PV anomalies are materially conserved, a property particularly useful in identifying and tracing the evolution of oceanic and meteorological disturbances. A second important property concerning $\mathrm{PV}$ is the possibility of recovering the pressure field, or the geopotential field, from the PV field given suitable boundary and balance conditions, a process usually referred to as PV inversion. These conservation and invertibility properties led to the concept of PV-thinking the approach that uses PV as a quantity 
from which the dynamical fields, including the advecting fluid velocity, may be obtained (Hoskins, McIntyre \& Robertson 1985). The conservation and invertibility properties are the main reason for the great usefulness of PV and why it is so frequently employed in theoretical and numerical studies (see reviews by Kurganskiy \& Tatarskaya 1987; Müller 1995) as well as in observational weather analysis and forecasting (Santurette \& Georgiev 2005).

Consistently with the PV thinking, a large number of processes in the atmosphere and oceans can be understood if these are considered to act as though they were composed of particles of PV (Bishop \& Thorpe 1994). In particular, the compact ball of constant PV, with spherical shape in the vertically stretched quasi-geostrophic (QG) space, provides a simple model for geophysical vortex flow (e.g. Holton 2004, $\S$ 6.3.4). The QG space has spatial dimensions $(x, y, \hat{z})$, where $(x, y, z)$, are the usual three-dimensional spatial physical dimensions and $\hat{z} \equiv c z$, where the Prandtl ratio $c \equiv N / f$ is the ratio between background constant Brunt-Väisälä $N$ and Coriolis $f$ frequencies. Numerical simulations of geostrophic turbulence support that the mean height-to-width aspect ratio of atmospheric and oceanic vortices is $\sim 0.8$ in the QG space (McWilliams, Weiss \& Yavneh 1999; Reinaud, Dritschel \& Koudella 2003). Geopotential solutions have been found via PV inversion of a spherical (in the QG space) PV vortex for the vortex interior and exterior regions under the QG (Thorpe \& Bishop 1994) and semigesotrophic (Thorpe \& Bishop 1995) approximations. Under the more general $f$-plane and Boussinesq approximations exact spherical interior solutions exist (Viúdez 2008) but exact exterior solutions are still lacking. In this work we provide elliptic and hyperbolic interior geopotential solutions obtained via PV inversion for a homogeneous distribution of PV. The elliptic solutions include the spherical ones as a particular case.

The basic dynamics, definitions of relevant quantities, and the PV inversion problem are first briefly introduced in $\S 2$. The PV inversion problem is then particularized in $\S 3$ to the case of steady and horizontal motion of an axisymmetrical three-dimensional vortex in which the centripetal acceleration plus the Coriolis acceleration equals the pressure anomaly gradient term. This balance is known as gradient wind or cyclogeostrophic balance. The analysis of the new elliptic and hyperbolic solutions is given in $\S 4$. First $(\S 4.1)$ it is found that vortex intensity and geometry of the interior geopotential surfaces may be completely specified by the vertical stratification $d_{z}$ and the absolute vertical vorticity $\tilde{\zeta}_{a} \equiv \tilde{\zeta}+1$. However, it turns out to be more convenient to specify the vortex using two parameters, namely, the PV in the vortex $(\Pi)$ and the squared aspect ratio (say $\mu$ ) between the horizontal and vertical semi-axes of the ellipsoidal geopotential surfaces. Thus, the family of geopotential solutions is parameterized by $\Pi$ and $\mu$ and the PV inversion task consists of relating $d_{z}$ and $\tilde{\zeta}_{a}$ to these parameters. The particular case of PV inversion in a spherical vortex is briefly considered ( $\$ 4.2)$ because it is simpler than the elliptic vortex, it is always statically and inertially stable for positive PV, and the geopotential solutions provide a check for the more general and complicated elliptic solutions. The equations for the elliptic and hyperbolic geometry vortex are then set out in $\S 4.3$. These equations are algebraic cubic equations admitting one real root or three distinct real roots depending on the values of their respective discriminant. The values of PV and geopotential surface aspect ratio that make the discriminants equal to zero separate the different regions in the PV-aspect ratio space that admit one or three real roots $(\S 4.4)$. It is found that there is always a critical aspect ratio beyond which the solutions are multi-valued. This aspect ratio depends on the PV value. Next, the complete vorticity 
and stratification solutions for the different regions are obtained and analysed $(\$ 4.5)$. Finally, concluding remarks are given in $\S 5$.

\section{Basic dynamics and PV}

We consider the isochoric (volume-preserving) motion of a stable stratified fluid, under the Boussinesq approximation, in a reference frame fixed at the surface of a sphere (e.g. the Earth) rotating with angular velocity $\boldsymbol{\Omega}$ with respect to an inertial frame, and whose vertical axis coincides with the direction of the sum of the gravity force (directed towards the centre of the sphere) and planetary centripetal acceleration (directed towards the axis of rotation). We assume the usual $f$-plane approximation (typical of mesoscale and synoptic-scale dynamics) which neglects locally the sphericity of the Earth and assumes plane gravity potential surfaces. Here $f$ is the vertical component of the planetary vorticity (or Coriolis parameter). It is convenient to introduce the density anomaly $\rho^{\prime}$ defined as

$$
\rho^{\prime}(\boldsymbol{x}, t) \equiv \rho(\boldsymbol{x}, t)-\varrho_{0} z-\rho_{0},
$$

where $\boldsymbol{x}=(x, y, z), \rho$ is the mass density, and $\rho_{0}>0$ and $\varrho_{0}<0$ are given constants that do not need to be specified under the Boussinesq approximation. We introduce the pressure anomaly $\mathscr{P}$ as the pressure $p$, minus the hydrostatic pressure due to a constant vertical density stratification

$$
\mathscr{P}(\boldsymbol{x}, t) \equiv p(\boldsymbol{x}, t)+g\left(\rho_{0}+\frac{1}{2} \varrho_{0} z\right) z
$$

where $g$ is the acceleration due to gravity and planetary centripetal acceleration. The Boussinesq approximation in the vertical component of the momentum equation is therefore

$$
-\frac{1}{\rho}\left(p_{z}+g \rho\right) \cong-\alpha_{0}\left(p_{z}+g \rho\right)=-\alpha_{0} \mathscr{P}_{z}-\alpha_{0} g \rho^{\prime},
$$

where $\alpha_{0} \equiv \rho_{0}^{-1}$ is a constant specific volume, and the subscript $z$ means the partial derivative with respect to $z$. The basic equations are the non-hydrostatic balance of linear momentum in a rotating frame under the $f$-plane and Boussinesq approximations, the mass conservation equation, and the isochoric condition,

$$
\begin{gathered}
\frac{\mathrm{d} \boldsymbol{u}_{h}}{\mathrm{~d} t}+f \hat{\boldsymbol{k}} \times \boldsymbol{u}_{h}=-\alpha_{0} \nabla_{h} \mathscr{P}, \\
\frac{\mathrm{d} w}{\mathrm{~d} t}=-\alpha_{0} \frac{\partial \mathscr{P}}{\partial z}-\alpha_{0} g \rho^{\prime}, \\
\frac{\mathrm{d} \rho}{\mathrm{d} t}+\rho \nabla \cdot \boldsymbol{u}=0, \\
\nabla \cdot \boldsymbol{u}=0 .
\end{gathered}
$$

As usual, $\mathrm{d}() / \mathrm{d} t \equiv \partial() / \partial t+\boldsymbol{u} \cdot \nabla()$ denotes the material time derivative in the rotating reference frame, and $\hat{\boldsymbol{k}}$ is the vertical unit vector. The unknowns are the threedimensional velocity field $\boldsymbol{u}=(u, v, w)$, the pressure anomaly $\mathscr{P}$, and the density anomaly $\rho^{\prime}$.

It is convenient to express $\rho$ in terms of the field $d$ defined by

$$
d \equiv\left(\rho-\rho_{0}\right) / \varrho_{0} ;
$$

$d(\boldsymbol{x}, t)$ represents the depth, or vertical location, that the isopycnal located at $\boldsymbol{x}$ at time $t$ has in the reference density configuration defined by $\rho_{0}+\varrho_{0} z$. Thus, the density field 
is expressed in terms of distances. The displacement $\mathscr{D}$ of isopycnals with respect to the reference density configuration is defined as

$$
\mathscr{D}(\boldsymbol{x}, t) \equiv z-d(\boldsymbol{x}, t) .
$$

$\mathscr{D}(\boldsymbol{x}, t)$ is the vertical displacement of the isopycnal currently located at $(\boldsymbol{x}, t)$ with respect to its reference position. The incompressibility condition $\mathrm{d} \rho / \mathrm{d} t=\mathrm{d} d / \mathrm{d} t=0$ is expressed in terms of $\mathscr{D}$ as

$$
\frac{\mathrm{d} \mathscr{D}}{\mathrm{d} t}=w .
$$

The vertical displacement of isopycnals $\mathscr{D}$ is related to $\rho$ and $\rho^{\prime}$ by

$$
N^{2}(\mathscr{D}(\boldsymbol{x}, t)-z)=g\left(\alpha_{0} \rho(\boldsymbol{x}, t)-1\right), \quad \mathscr{D}=-\frac{\rho^{\prime}}{\varrho_{0}},
$$

where $N^{2} \equiv-\alpha_{0} g \varrho_{0}$ is the square of the constant background Brunt-Väisälä frequency. Thus, the buoyancy term $\alpha_{0} g \rho^{\prime}$ in $(2.4 b)$ can be replaced with $N^{2} \mathscr{D}$. The relation between the total $\mathscr{N}(\boldsymbol{x}, t)$ and constant background $N$ Brunt-Väisälä frequencies is

$$
\mathscr{N}^{2}(\boldsymbol{x}, t)=N^{2}\left(1-\frac{\partial \mathscr{D}}{\partial z}(\boldsymbol{x}, t)\right) .
$$

We note that static instability $(\partial \rho / \partial z>0)$ occurs when the stratification number $\partial \mathscr{D} / \partial z>1$.

For any quantity $\chi$ let $\tilde{\chi} \equiv \chi / f$. The rate of change of $\nabla \mathscr{D}$, obtained from (2.7), and the rate of change of the dimensionless relative vorticity $\tilde{\boldsymbol{\omega}} \equiv \nabla \times \tilde{\boldsymbol{u}}=(\tilde{\xi}, \tilde{\eta}, \tilde{\zeta})$, obtained from $(2.4 a, b)$ imply the material conservation of PV anomalies

$$
\frac{\mathrm{d} \varpi}{\mathrm{d} t}=0
$$

where

$$
\varpi \equiv \Pi-1 \equiv(\tilde{\boldsymbol{\omega}}+\hat{\boldsymbol{k}}) \cdot \nabla d-1=\tilde{\zeta}-\frac{\partial \mathscr{D}}{\partial z}-\tilde{\boldsymbol{\omega}} \cdot \nabla \mathscr{D}
$$

is the dimensionless $P V$ density anomaly. Since the flow is isochoric, both PV density ( $\Pi)$ and specific PV $(\Pi / \rho)$ are materially conserved (see Viúdez 2001, for the rationale for this nomenclature). If the spatial distribution of PV anomaly $\varpi(x)$ is known, and considering the vorticity $\tilde{\boldsymbol{\omega}}\{\mathscr{P}(\boldsymbol{x})\}$ and the vertical displacement $\mathscr{D}\{\mathscr{P}(\boldsymbol{x})\}$ as functions of the spatial derivatives of the unknown pressure anomaly $\mathscr{P}$, the PV definition (2.11) becomes an equation for $\mathscr{P}(\boldsymbol{x})$. This is called the PV inversion problem, and is solved in this paper in the particular case of ellipsoidal and hyperbolic

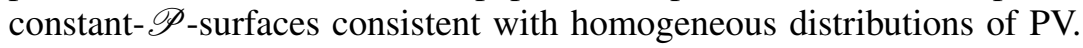

\section{Gradient balance vortex}

In this section we consider the steady and horizontal motion of a three-dimensional vortex in which the centripetal acceleration plus the Coriolis acceleration equals the pressure-anomaly gradient term. This balance is commonly referred to as gradient wind or cyclo-geostrophic balance. We use cylindrical coordinates $(r, \phi, z)$, where $r^{2}(x, y) \equiv x^{2}+y^{2}$. The dependent variables are the transverse (azimuthal) velocity $v$, $\mathscr{D}$ and $\Phi$ (the scaled pressure anomaly, defined below), since the radial and vertical 
velocities $u=w=0$. The flow satisfies (2.4) which in this case is reduced to the balance of momentum along $r$, and the hydrostatic condition,

$$
\begin{gathered}
\frac{\tilde{v}^{2}}{r}+\tilde{v}=\Phi_{r}, \\
0=\Phi_{z}+c^{2} \mathscr{D},
\end{gathered}
$$

where we recall that $c \equiv N / f$ and we have defined the scaled pressure anomaly

$$
\Phi(r, z) \equiv \frac{\alpha_{0}}{f^{2}} \mathscr{P}(r, z)
$$

The solutions of $\tilde{v}$ and $\mathscr{D}$ in terms of $\Phi(r, z)$ are

$$
\tilde{v}=\frac{r}{2}\left( \pm \sqrt{1+4 \frac{\Phi_{r}}{r}}-1\right), \quad \mathscr{D}=-\epsilon^{2} \Phi_{z},
$$

where $\epsilon \equiv c^{-1}=f / N$. The positive root in the expression for $\tilde{v}$ above should be taken if one desires to ensure that $\tilde{v}=0$ when $\Phi_{r}=0$, as is usually done when the gradient balance condition is assumed. We continue considering both solutions, however, for the sake of generality. It is assumed therefore, for $(3.3 a)$ to have real solutions, that $1+4 \Phi_{r} / r \geqslant 0$. In the particular case $1+4 \Phi_{r} / r=0$ the flow $\tilde{v}=-r / 2$ is barotropic (independent of $z$ ) and the relative vorticity $\tilde{\zeta}=-1$, so that $\zeta=-f$.

In this three-dimensional case the PV anomaly definition (2.11) of the $\hat{\boldsymbol{k}}$-axis symmetrical vortex can be written in cylindrical coordinates $(r, z)$ as

$$
\Pi-1=\varpi=\tilde{\zeta}-\mathscr{D}_{z}-\tilde{\xi} \mathscr{D}_{r}-\tilde{\zeta} \mathscr{D}_{z},
$$

where $\tilde{\xi}=-\tilde{v}_{z}$ is the radial component of vorticity, $\tilde{\zeta}_{a} \equiv \tilde{\zeta}+1$ is the dimensionless absolute vertical vorticity, and $d_{z}$ is the dimensionless vertical stratification. In terms of the pressure anomaly $\Phi$ the contributions to $\varpi$ are

$$
\begin{aligned}
\tilde{\zeta} & =\frac{1+3 \Phi_{r} / r+\Phi_{r r}}{ \pm \sqrt{1+4 \Phi_{r} / r}}-1, \\
\mathscr{D}_{z} & =-\Phi_{\hat{z} \hat{z}}, \\
\tilde{\xi} \mathscr{D}_{r} & =\frac{\Phi_{r \hat{z}}^{2}}{ \pm \sqrt{1+4 \Phi_{r} / r}}, \\
\tilde{\zeta} \mathscr{D}_{z} & =\left(1-\frac{1+3 \Phi_{r} / r+\Phi_{r r}}{ \pm \sqrt{1+4 \Phi_{r} / r}}\right) \Phi_{\hat{z} \hat{z}},
\end{aligned}
$$

where we recall that $\hat{z} \equiv c z$. Above we assume also that $1+4 \Phi_{r} / r \neq 0$, so that $\zeta \neq-f$.

Suitably combining the terms above leads to the relation between PV and pressure gradient,

$$
\pm \Pi \sqrt{1+4 \frac{\Phi_{r}}{r}}=1+\Phi_{r r}+\Phi_{\hat{z} \hat{z}}+\Phi_{r r} \Phi_{\hat{z} \hat{z}}-\Phi_{r \hat{z}}^{2}+3 \frac{\Phi_{r}}{r}\left(1+\Phi_{\hat{z} \hat{z}}\right),
$$

which is a Monge-Ampère equation for the unknown $\Phi(r, \hat{z})$. We see that, with regard to the PV inversion, selecting sign in front of the square root for $\tilde{v}$ in $(3.3 a)$ is equivalent to choosing the sign of $\Pi$. Therefore only one of these two conditions needs to be considered in the analysis. Here we consider the possibility of negative PV though we will see below that, due to symmetry relations, equations for $\Pi<0$ need 
not be explicitly written down. In the following section we find elliptic and hyperbolic solutions of (3.6) valid for the interior of a constant-PV vortex, and therefore valid at the centre of the PV vortex where $(r, \hat{z})=(0,0)$. Closed-form solutions for the vortex exterior where $\varpi=0$ and such that $\Phi \rightarrow 0$ as $r^{2}+\hat{z}^{2} \rightarrow \infty$ are more difficult to obtain. Finding these exterior solutions is an important theoretical challenge which is left for future work.

\section{Elliptic and hyperbolic interior solutions of a piecewise-constant symmetric PV vortex}

\subsection{The PV and aspect ratio description}

We seek elliptic or hyperbolic solutions of the PV inversion equation (3.6) of the form

$$
\Phi(r, \hat{z})=g_{1}(\Pi) r^{2}+g_{2}(\Pi) \hat{z}^{2},
$$

valid for the interior of the PV vortex with homogeneous $\Pi$. The above solution satisfies (3.6) as long as

$$
\tilde{\zeta}_{a}= \pm \sqrt{1+8 g_{1}}, \quad d_{z}=1+2 g_{2},
$$

and therefore (4.1) can be written in terms of $\tilde{\zeta}_{a}$ and $d_{z}$ as

$$
\Phi(r, \hat{z})=\frac{\tilde{\zeta}_{a}^{2}-1}{8} r^{2}+\frac{d_{z}-1}{2} \hat{z}^{2},
$$

where $\tilde{\zeta}_{a}$ and $d_{z}$ are constants independent of $(r, \hat{z})$. We note that the pair $\left\{\tilde{\zeta}_{a}, d_{z}\right\}$ specify both the geometry and the constant PV anomaly of the vortex, and therefore completely characterize the vortex. However the vortex geometry can be more directly specified by any of the aspect ratios

$$
\mu \equiv \frac{g_{2}}{g_{1}}, \quad v \equiv \frac{g_{1}}{g_{2}}=\frac{1}{\mu} .
$$

Ratios $\mu$ and $v$ are the (squared) width-to-height and height-to-width aspect ratios, respectively. Spheroid vortices have $\operatorname{sgn}\left(g_{1}\right)=\operatorname{sgn}\left(g_{2}\right)$ while hyperbolic vortices have $\operatorname{sgn}\left(g_{1}\right)=-\operatorname{sgn}\left(g_{2}\right)$. Prolate spheroid vortices have vertical and horizontal semi-axes $g_{2}^{-1 / 2}>g_{1}^{-1 / 2}$ and therefore $v>1$ and $\mu<1$, while oblate spheroid vortices have $v<1$ and $\mu>1$. This nomenclature is always applied here in the vertically stretched QG space of coordinates $(r, \hat{z})$. Thus, a spheroid vortex in the QG space with aspect ratios $\mu$ and $\nu$ is a spheroid of aspect ratios $\mu^{\prime}=c \mu$ and $v^{\prime}=v / c$, respectively, in the physical space of coordinates $(r, z)$.

In terms of $\left\{\tilde{\zeta}_{a}, d_{z}\right\}$ the PV definition (3.4) is simply

$$
\Pi=\tilde{\zeta}_{a} d_{z},
$$

and the aspect ratios

$$
\mu\left(\tilde{\zeta}_{a}, d_{z}\right)=4 \frac{d_{z}-1}{\tilde{\zeta}_{a}^{2}-1}, \quad v\left(\tilde{\zeta}_{a}, d_{z}\right)=\frac{\tilde{\zeta}_{a}^{2}-1}{4\left(d_{z}-1\right)} .
$$

We have therefore two descriptions, depending on whether the independent variables are $\left\{\tilde{\zeta}_{a}, d_{z}\right\}$ or $\{\Pi, \mu \mid v\}$. We use hereinafter the $\{\Pi, \mu \mid v\}$ description because the complete dynamical characteristics of the flow are given more directly by the PV content and PV geometry of the vortex. In this description, $\tilde{\zeta}_{a}$ and $d_{z}$ are considered 
as functions of $\{\Pi, \mu\}$ and $\{\Pi, \nu\}$, respectively. Two aspect ratio parameters are used, $\mu$ and $\nu$, instead of only one of them as might seem more simple, to avoid, as explained later, mathematical singularities in the relevant equations.

\subsection{Spherical geometry}

It is useful first to consider briefly the case of spherical geometry in the QG space. In this case the aspect ratios $\mu=v=1$ and (4.6) reduces to

$$
\tilde{\zeta}_{a}^{2}=4 d_{z}-3
$$

Thus, spherical vortices with homogeneous $\mathrm{PV}$ require a stratification $d_{z} \geqslant 3 / 4$, and therefore $\mathscr{D}_{z}=1-d_{z} \leqslant 1 / 4$, that is, they are always statically stable $\left(\mathscr{D}_{z}<1\right)$. In the spherical case, and using (4.5), we obtain the vertical vorticity and vertical stratification equations

$$
\tilde{\zeta}_{a}^{3}+3 \tilde{\zeta}_{a}-4 \Pi=0, \quad 4 d_{z}^{3}-3 d_{z}^{2}-\Pi^{2}=0
$$

The discriminants $D_{\zeta}$ and $D_{d}$ of these equations (see e.g. Woan 2003, p. 51) are, respectively,

$$
D_{\zeta}(\Pi)=1+4 \Pi^{2}, \quad D_{d}(\Pi)=\Pi^{2}\left(1+4 \Pi^{2}\right) / 2^{8},
$$

which are always positive and therefore their real solutions are univalued. The solutions of (4.8) are

$$
\begin{gathered}
\tilde{\zeta}_{a}(\Pi)=\sqrt[3]{2 \Pi+\sqrt{4 \Pi^{2}+1}}+\sqrt[3]{2 \Pi-\sqrt{4 \Pi^{2}+1}} \\
d_{z}(\Pi)=\frac{1}{4} \sqrt[3]{8 \Pi^{2}+1+4 \Pi \sqrt{4 \Pi^{2}+1}}+\frac{1}{4} \sqrt[3]{8 \Pi^{2}+1-4 \Pi \sqrt{4 \Pi^{2}+1}}+\frac{1}{4}
\end{gathered}
$$

We notice, consistently with (4.8), the symmetry relations $\tilde{\zeta}_{a}(-\Pi)=-\tilde{\zeta}_{a}(\Pi)$ and $d_{z}(-\Pi)=d_{z}(\Pi)$.

\subsection{Elliptic and hyperbolic geometry}

We turn now to the general elliptic-hyperbolic case. From (4.6), $\tilde{\zeta}_{a}$ and $d_{z}$ satisfy the cubic equations

$$
\tilde{\zeta}_{a}^{3}+(4 v-1) \tilde{\zeta}_{a}-4 v \Pi=0, \quad d_{z}^{3}+\left(\frac{\mu}{4}-1\right) d_{z}^{2}-\frac{\mu}{4} \Pi^{2}=0 .
$$

These equations are the vertical vorticity and vertical stratification PV inversion equations, hereinafter for short just referred to as the vorticity and stratification equations. We next solve these cubic equations and analyse their solutions.

We use the descriptions $\{\Pi, \nu\}$ for $\tilde{\zeta}_{a}$ and $\{\Pi, \mu\}$ for $d_{z}$ in (4.12). Thus we employ the two parameters $\{\mu, \nu\}$ instead of using only one of them, to avoid employing $\mu^{-1}$ or $v^{-1}$, and therefore avoiding the appearance of singularities in the $\tilde{\zeta}_{a}$ or $d_{z}$ distributions as $\mu \rightarrow 0$ or $v \rightarrow 0$. The inconvenience, however, derived from using these two descriptions, $(\Pi, v)$ for symbol $\tilde{\zeta}_{a}$ and $(\Pi, \mu)$ for symbol $d_{z}$, is that some care must be taken when an equality involving values is expressed, using the same symbols, in terms of functions. For example, the equality (4.5) involving values must be now expressed using the composition of functions as $\Pi=\tilde{\zeta}_{a}(\Pi, v) d_{z}(\Pi, 1 / v)$ or as $\Pi=\tilde{\zeta}_{a}(\Pi, 1 / \mu) d_{z}(\Pi, \mu)$.

Clearly in (4.12) simplification of terms is possible by introducing new symbols for $4 v$ and its inverse $\mu / 4$. We, however, prefer to use symbols $v$ and $\mu$ because these have a direct geometrical meaning representing the ratio between factors in (4.3). 
Our analysis is restricted here to the range $\Pi \geqslant 0$. Solutions of the vertical vorticity and vertical stratification equations for $\Pi<0$ can be obtained from the respective solutions $\tilde{\zeta}_{a}(\Pi, v)$ and $d_{z}(\Pi, \mu)$ for $\Pi>0$ by noticing that the vorticity equation (4.12a) is antisymmetric with respect to $\Pi$, so that $\tilde{\zeta}_{a}(-\Pi, v)=-\tilde{\zeta}_{a}(\Pi, v)$, and that the vertical stratification equation $(4.12 b)$ is symmetric with respect to $\Pi$, so that $d_{z}(-\Pi, \mu)=d_{z}(\Pi, \mu)$. These symmetry relations are consistent with the PV equality (4.5). Note however that, due to (4.5), negative PV always implies one and only one type of instability, that is, inertial instability $\left(\tilde{\zeta}_{a}<0\right)$ or static instability $\left(d_{z}<0\right)$.

\subsection{The different regions in the $(\Pi, \mu \mid \nu)$-space based on the discriminants of the vorticity and stratification equations}

In order to find the solutions of the vorticity and stratification equations (4.12) we obtain first their respective discriminants. If the discriminant $D>0$ there is only one real root, while if $D<0$ there are three distinct real roots. In the case $D=0$ there are three real roots but at least two are equal.

The discriminant of the vorticity equation $(4.12 a)$ is

$$
D_{\zeta}(\Pi, v)=4 v^{2} \Pi^{2}+\left(\frac{4 v-1}{3}\right)^{3} .
$$

In the spherical case $v=1$ and $D_{\zeta}(\Pi, 1)$ equals $D_{\zeta}(\Pi)$ given by $(4.9 a)$. In order to analyse the solutions $\tilde{\zeta}_{a i}, i=1,2, \ldots$, it is convenient to divide the parametric space $\{\Pi \geqslant 0, v\}$ into regions or domains defined by the sign of $D_{\zeta}(\Pi, v)$. From the condition $D_{\zeta}\left(\Pi_{\zeta}, v\right)=0$, the solution curve $\Pi_{\zeta}(v)$ separating these domains is

$$
\Pi_{\zeta}(v)=\frac{1}{ \pm 6 v} \sqrt{\frac{(1-4 v)^{3}}{3}} \quad \text { where }(+): 0<v \leqslant 1 / 4 \text { and }(-): v<0 .
$$

Function $\Pi_{\zeta}(v)$ is represented in figure $1 . \Pi_{\zeta}(v)$ has a minimum at $v=1 / 4$ where $\Pi_{\zeta}=0$, it has a relative minimum at $v=-1 / 2$ where $\Pi_{\zeta}=1$ (point $\mathrm{P}$ in figure 1), and $\Pi_{\zeta} \rightarrow \infty$ as $v \rightarrow 0^{ \pm}$and $v \rightarrow-\infty$.

It is useful also to obtain the inverse function $v(\Pi)$ satisfying $D_{\zeta}(\Pi, v)=0$. This function obeys a cubic equation and, as can be inferred from figure 1 , is multi-valued for $\Pi \geqslant 1$ and therefore is more complicated than $\Pi_{\zeta}(v)$. The explicit solutions $v_{i}(\Pi)$ are

$$
\begin{aligned}
v_{1}(\Pi)= & \frac{3}{2^{4}} \Pi^{2 / 3}\left[\sqrt[3]{9 \Pi^{2}\left(4-3 \Pi^{2}\right)-8+8 \sqrt{1-\Pi^{2}}}\right. \\
& \left.+\sqrt[3]{9 \Pi^{2}\left(4-3 \Pi^{2}\right)-8-8 \sqrt{1-\Pi^{2}}}\right]+\frac{4-9 \Pi^{2}}{2^{4}}, \quad 0 \leqslant \Pi \leqslant 1 \\
v_{2}(\Pi)= & \frac{3}{8}|\Pi| \sqrt{9 \Pi^{2}-8} \cos \left(\frac{\theta_{\nu}(\Pi)}{3}\right)+\frac{4-9 \Pi^{2}}{2^{4}}, \quad \Pi \geqslant 1 \\
v_{3}(\Pi)= & -\frac{3}{8}|\Pi| \sqrt{9 \Pi^{2}-8} \cos \left(\frac{\theta_{\nu}(\Pi)+\pi}{3}\right)+\frac{4-9 \Pi^{2}}{2^{4}}, \quad \Pi \geqslant 1 \\
v_{4}(\Pi)= & -\frac{3}{8}|\Pi| \sqrt{9 \Pi^{2}-8} \cos \left(\frac{\theta_{\nu}(\Pi)-\pi}{3}\right)+\frac{4-9 \Pi^{2}}{2^{4}}, \quad \Pi \geqslant 1
\end{aligned}
$$




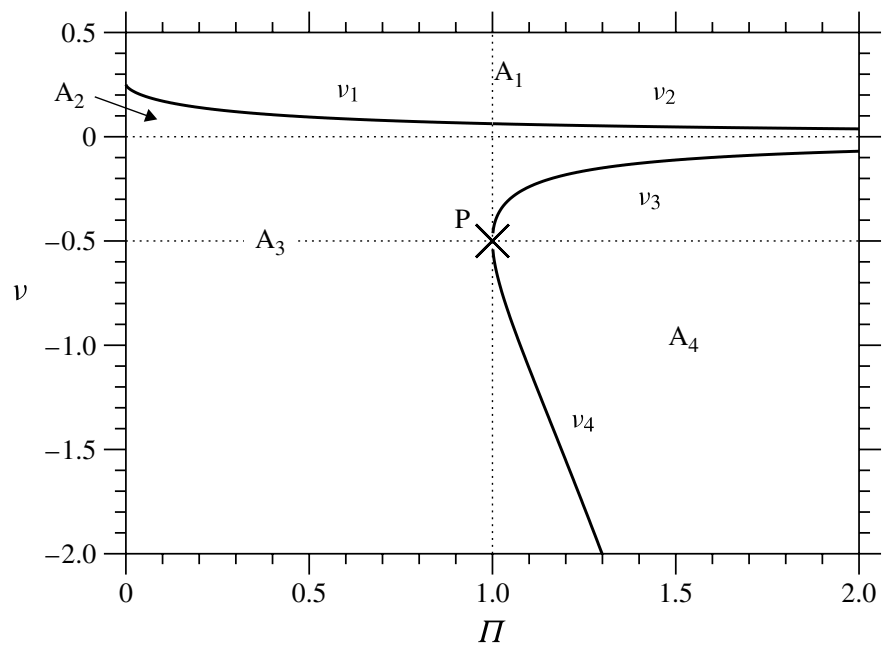

FIGURE 1. Solution $\Pi_{\zeta}(v)$ as given by (4.14) or, alternatively, the inverse solutions $v_{i}(\Pi), i=1, \ldots, 4$, as given by (4.15). Note that $v_{1}(1)=v_{2}(1)=1 / 16$, whereas at the relative minimum point $\mathrm{P}$ of $\Pi_{\zeta}(v)$, shown with symbol $\times$, we have $v_{3}(1)=v_{4}(1)=-1 / 2$.

where

$$
\theta_{v}(\Pi) \equiv \arccos \frac{9 \Pi^{2}\left(4-3 \Pi^{2}\right)-8}{|\Pi|\left(9 \Pi^{2}-8\right)^{3 / 2}}
$$

We note that as $\Pi \rightarrow \infty$ we have $\theta_{v} \rightarrow \pi$ and therefore $v_{2} \rightarrow 0, \nu_{3} \rightarrow 0$, and $v_{4}(\Pi) \sim-9 \Pi^{2} / 2^{4}$ as seen in figure 1. At $\Pi=1$ we have $v_{1}(1)=v_{2}(1)=1 / 2^{4}$, and the relative minimum at point $\mathrm{P}$ in figure 1 is $(\Pi, v)=\left(1, v_{3}(1)\right)=\left(1, v_{4}(1)\right)=$ $(1,-1 / 2)$.

Regions $A_{1}$ and $A_{4}$ are the domain of the unique solution $\tilde{\zeta}_{a 1}$, while region $A_{2} \cup A_{3}$ is the domain of the three solutions $\left\{\tilde{\zeta}_{a 2}, \tilde{\zeta}_{a 3}, \tilde{\zeta}_{a 4}\right\}$ (see figure 2 for solutions $\tilde{\zeta}_{a 1}$ and $\left.\tilde{\zeta}_{a 2}\right)$. This latter domain is composed of regions $\mathrm{A}_{2}$, defined for $v \geqslant 0$ and $\mathrm{A}_{3}$, defined for $v<0$. Since $D(\Pi, v=0) \neq 0$ the solutions $\left\{\tilde{\zeta}_{a 2}, \tilde{\zeta}_{a 3}, \tilde{\zeta}_{a 4}\right\}$ are continuous at $v=0$ so that region $\mathrm{A}_{2} \cup \mathrm{A}_{3}$ is a unique domain. However it is convenient, for a comparison with the analysis of the stratification solutions $d_{z i}(\Pi, \mu)$ carried out in the next section, to consider this latter region as composed of the two regions $\mathrm{A}_{2}$ and $\mathrm{A}_{3}$.

The domain of the unique solution $\tilde{\zeta}_{a 1}$ is regions $\mathrm{A}_{1}$ and $\mathrm{A}_{4}$ where $\Pi>\Pi_{\zeta}$. Region $A_{1}$ is the domain of elliptic vortices $(v>0)$, and $A_{4}$ is the domain of hyperbolic vortices $(v<0)$. Region $\mathrm{A}_{2} \cup \mathrm{A}_{3}$, where $\Pi \leqslant \Pi_{\zeta}$, is the domain of the three real solutions $\left\{\tilde{\zeta}_{a 2}, \tilde{\zeta}_{a 3}, \tilde{\zeta}_{a 4}\right\}$.

We obtain next the different domains, in the configuration $(\Pi, \mu)$, for the stratification $d_{z}$. The discriminant $D_{d}(\Pi, \mu)$ of the stratification equation $(4.12 b)$ is

$$
D_{d}(\Pi, \mu)=\frac{\mu \Pi^{2}}{2^{6}}\left[\mu \Pi^{2}+\frac{1}{4}\left(\frac{4-\mu}{3}\right)^{3}\right]
$$




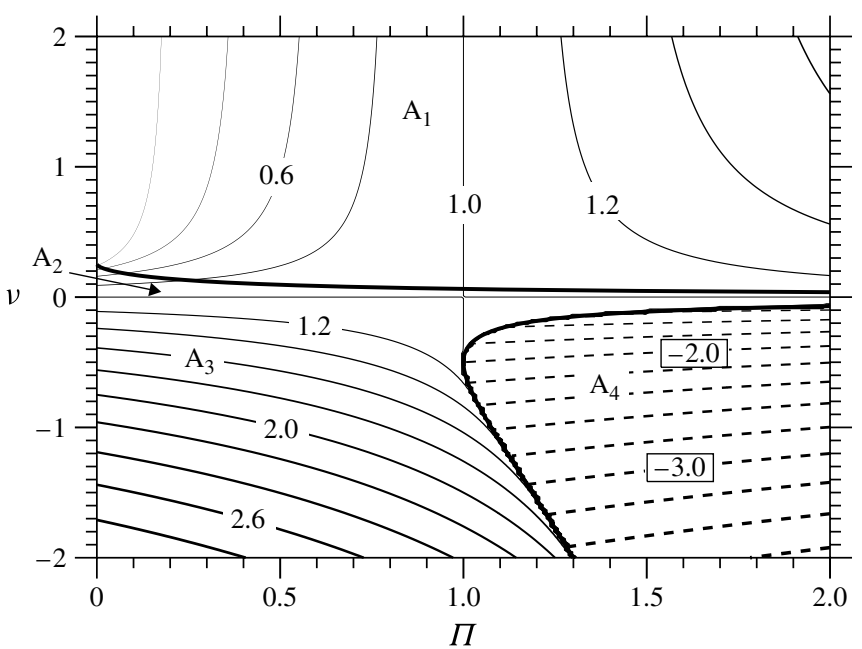

FIGURE 2. Solutions $\tilde{\zeta}_{a 1}(\Pi, v)$ in regions $\mathrm{A}_{1}$ and $\mathrm{A}_{4}$, and $\tilde{\zeta}_{a 2}(\Pi, v)$ in region $\mathrm{A}_{2} \cup \mathrm{A}_{3}$. The curve $\Pi_{\zeta}(v)$ (continuous thick line) is included. Contour interval $\Delta=0.2$. The four regions $\mathrm{A}_{1}-\mathrm{A}_{4}$ are shown. Note that the union of functions $\tilde{\zeta}_{a 1}$ and $\tilde{\zeta}_{a 2}$ is continuous on the branch $\Pi_{\zeta}(v>0)$ but discontinuous on $\Pi_{\zeta}(v<0)$. Absolute vorticity $\tilde{\zeta}_{a 1} \rightarrow \Pi$ as $v \rightarrow \infty$.

In the spherical case $\mu=1$ and $D_{d}(\Pi, 1)$ equals $D_{d}(\Pi)$ given by (4.9b). Thus $D_{d}(\Pi, \mu)=0$ when $\Pi=0$ or $\mu=0$, or for $\mu \neq 0$ when $\Pi=\Pi_{d}(\mu)$ is given by

$$
\Pi_{d}(\mu)=\frac{1}{6} \sqrt{\frac{(\mu-4)^{3}}{3 \mu}},
$$

which is valid for $\mu \geqslant 4$ and $\mu<0$. Function $\Pi_{d}(\mu)$ is shown in figure $3 . \Pi_{d}(\mu)$ has a minimum at $\mu=4$ where $\Pi_{d}=0$, a relative minimum at $\mu=-2$ where $\Pi_{d}=1$ (point $\mathrm{P}$ in figure 3), and $\Pi_{d} \rightarrow \infty$ as $\mu \rightarrow \pm \infty$ and $\mu \rightarrow 0^{-}$. The domain of the unique solution $d_{z 1}$ is regions $\mathrm{B}_{1}$ and $\mathrm{B}_{4}$ where $\Pi>\Pi_{d}$, region $\mathrm{B}_{1}$ in the case of elliptic vortices $(\mu>0)$, and $\mathrm{B}_{4}$ in the case of hyperbolic vortices $(\mu<0)$. Regions $\mathrm{B}_{2}$ and $\mathrm{B}_{3}$, where $\Pi \leqslant \Pi_{d}$, are the domain of the three real solutions $\left\{d_{z 1}, d_{z 2}, d_{z 3}\right\}$. The subindices of these regions in the $(\Pi, \mu)$-space have been chosen so that they equal the corresponding regions in the $(\Pi, v)$-space, symbolically $\mathrm{A}_{i} \leftrightarrow \mathrm{B}_{i}$. This correspondence between different $\mathrm{A}_{i}$ and $\mathrm{B}_{i}$ regions is due to (4.5), which implies that to every solution $\tilde{\zeta}_{a i}(\Pi, v), i=\{1,2,3,4\}$, corresponds a solution $d_{z j}(\Pi, 1 / \nu)$ for some $j=\{1,2,3,4\}$. Thus, those regions in the parametric space $(\Pi, v)$ allowing three real solutions $\tilde{\zeta}_{a i}(\Pi, v)$ must also allow three real solutions $d_{z j}(\Pi, 1 / v)$. This also implies that $\Pi_{\zeta}(v)=\Pi_{d}(1 / v)=\Pi_{d}(\mu)=\Pi_{\zeta}(1 / \mu)$, as can be verified from (4.14) and (4.17). For this reason the minimum of $\Pi_{\zeta}$ at $v=1 / 4$ corresponds to the minimum of $\Pi_{d}$ at $\mu=1 / \nu=4$, and the relative minimum (point $\mathrm{P}$ in figures 1 and 3 ) occurs at $\nu=-1 / 2$ and at $\mu=1 / \nu=-2$.

The inverse solutions $\mu_{i}(\Pi)$ of (4.17) are

$$
\begin{aligned}
& \mu_{1}(\Pi)=6 \Pi^{2 / 3}\left(\sqrt[3]{1+\sqrt{1-\Pi^{2}}}+\sqrt[3]{1-\sqrt{1-\Pi^{2}}}\right)+4, \quad 0 \leqslant \Pi<1 \\
& \mu_{2}(\Pi)=4\left(1+3 \Pi \cos \frac{\theta_{\mu}(\Pi)}{3}\right), \quad 1 \leqslant \Pi
\end{aligned}
$$




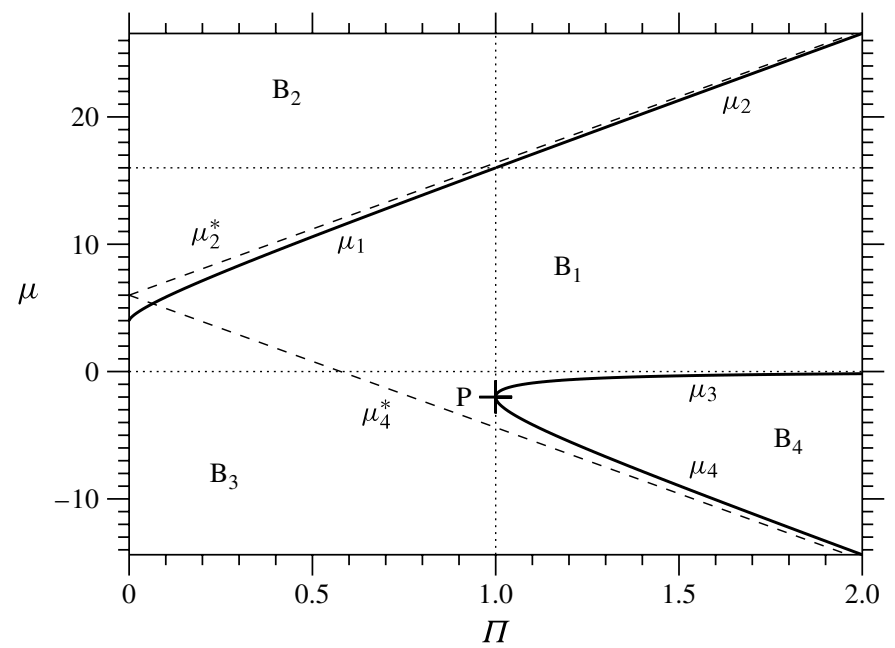

FIGURE 3. Solutions $\mu_{i}(\Pi) i=1, \ldots, 4$, and the asymptotes $\mu_{2}^{*}(\Pi)$ and $\mu_{4}^{*}(\Pi)$. At $\Pi=1$ functions $\mu_{1}$ and $\mu_{2}$ have a common value $\mu_{1}(1)=\mu_{2}(1)=16$, while $\mu_{3}$ and $\mu_{4}$ have a common value $\mu_{3}(1)=\mu_{4}(1)=-2$ where the relative minimum point $\mathrm{P}$ of $\Pi_{d}(\mu)$ is shown with the symbol + . The asymptotes intersect at $\Pi=0$ where $\mu_{2}^{*}(0)=\mu_{4}^{*}(0)=6$.

$$
\begin{array}{ll}
\mu_{3}(\Pi)=4\left(1-3 \Pi \cos \frac{\theta_{\mu}(\Pi)+\pi}{3}\right), & 1 \leqslant \Pi \\
\mu_{4}(\Pi)=4\left(1-3 \Pi \cos \frac{\theta_{\mu}(\Pi)-\pi}{3}\right), & 1 \leqslant \Pi
\end{array}
$$

where

$$
\theta_{\mu}(\Pi) \equiv \arccos \frac{1}{\Pi}
$$

Surprisingly, the phase $\theta_{\mu}(\Pi)$ is simply the arccos of $\Pi^{-1}$. The asymptotes as $\Pi \rightarrow \infty$ are $\mu_{2}^{*}(\Pi)=6+6 \sqrt{3} \Pi, \mu_{3}^{*}(\Pi)=0$ and $\mu_{4}^{*}(\Pi)=6-6 \sqrt{3} \Pi$, which have been obtained using the approximation $\arccos (1 / \Pi) \sim \pi / 2-1 / \Pi$.

\subsection{The vorticity and stratification solutions}

The unique solution $\tilde{\zeta}_{a 1}$, obtaining by solving the vorticity equation $(4.12 a)$, is

$$
\begin{aligned}
\tilde{\zeta}_{a 1}(\Pi, v)= & \sqrt[3]{2 v \Pi+\sqrt{4 v^{2} \Pi^{2}+\left(\frac{4 v-1}{3}\right)^{3}}} \\
& +\sqrt[3]{2 v \Pi-\sqrt{4 v^{2} \Pi^{2}+\left(\frac{4 v-1}{3}\right)^{3}}} .
\end{aligned}
$$

We note that for a spherical vortex in the QG space $v=1$ and $\tilde{\zeta}_{a 1}(\Pi, 1)$, given by (4.19), equals the vertical vorticity of the spherical vortex $\tilde{\zeta}_{a}(\Pi)$ given by (4.10). In the special case of oblate vortices with $v=1 / 4$ we have $\tilde{\zeta}_{a 1}(\Pi, 1 / 4)=\Pi^{1 / 3}$, as can be directly obtained from $(4.12 a)$. Function $\tilde{\zeta}_{a 1}$ is clearly antisymmetric, satisfying $\tilde{\zeta}_{a 1}(-\Pi, v)=-\tilde{\zeta}_{a 1}(\Pi, v)$. This solution is shown in figure 2 in regions $\mathrm{A}_{1}$ and $\mathrm{A}_{4}$. 
The unique solution $d_{z 1}$ of the stratification equation $(4.12 b)$ is

$$
\begin{aligned}
d_{z 1}(\Pi, \mu)= & \frac{1}{4} \sqrt[3]{8 \mu \Pi^{2}+\left(\frac{4-\mu}{3}\right)^{3}+4 \Pi \sqrt{4 \mu^{2} \Pi^{2}+\mu\left(\frac{4-\mu}{3}\right)^{3}}} \\
& +\frac{1}{4} \sqrt[3]{8 \mu \Pi^{2}+\left(\frac{4-\mu}{3}\right)^{3}-4 \Pi \sqrt{4 \mu^{2} \Pi^{2}+\mu\left(\frac{4-\mu}{3}\right)^{3}}+\frac{1}{4}\left(\frac{4-\mu}{3}\right)} .
\end{aligned}
$$

For a spherical vortex in the QG space $\mu=1$ and $d_{z 1}(\Pi, 1)$ given by (4.20) equals the vertical vorticity of the spherical vortex $d_{z}(\Pi)$ given by (4.11). Function $d_{z 1}(\Pi, \mu)$ is symmetric with respect to $\Pi$ since $d_{z 1}(-\Pi, \mu)=d_{z 1}(\Pi, \mu)$. In the special case of oblate vortices with $\mu=4$ expression (4.20) predicts that the vertical stratification has a simple dependence on $\mathrm{PV}, d_{z 1}(\Pi, 4)=\Pi^{2 / 3}$, which is consistent with $\tilde{\zeta}_{a 1}(\Pi, 1 / 4)=\Pi^{1 / 3}$ and (4.5). This result can be directly obtained from $(4.12 b)$. Also, when $\mu=0$, the PV ellipsoid transforms into a PV vertical cylinder, the stratification $d_{z 1}(\Pi, 0)=1$, the vertical displacement of isopycnals $\mathscr{D}$ is independent of $z$ inside the vertical cylinder, and $\tilde{\zeta}_{a 1}(\Pi, \infty)=\Pi$, so that the relative vertical vorticity $\tilde{\zeta}_{1}(\Pi, \infty)=\varpi$, and the flow becomes two-dimensional in the sense of being independent of $z$.

Region 1 (i.e. $A_{1}$, or equivalently $B_{1}$ ) is the region of balanced dynamics, where the vortices are elliptic, the relative vorticity $\tilde{\zeta}=\tilde{\zeta}_{a 1}-1$ has the same sign as the PV anomaly $\varpi=\Pi-1$, while the displacement stratification $\mathscr{D}_{z}=1-d_{z}$ has a different sign from that of the PV anomaly. Thus, for example, a subsurface anticyclonic vortex centred vertically at $z_{0}$ has negative relative vorticity and deepening isopycnals $(\mathscr{D}<0)$ below $z_{0}$, flat isopycnals at $z_{0}($ i.e. $\mathscr{D}=0)$, and rising isopycnals above $z_{0}$ (i.e. $\mathscr{D}>0$ ), so that $\mathscr{D}_{z}>0$ in the vortex. In this region zero PV anomalies $(\varpi=0, \Pi=1)$ imply no relative vorticity $\left(\tilde{\zeta}=0, \tilde{\zeta}_{a}=1\right.$, figure 2$)$ and no displacement stratification $\left(\mathscr{D}_{z}=0, d_{z}=1\right.$, figure 4$)$. Assuming no flow $(v=0)$ and flat isopycnals $(\mathscr{D}=0)$ on the domain boundaries, the condition $\varpi=0$ implies a state of rest in the rotating reference frame (so that $\Phi=0$ ) with flat isopycnals. In the upper limit of oblate vortices $(v=0)$ there is no relative vorticity $\left(\tilde{\zeta}_{a 1}=1\right)$ and, due to $(4.5)$, there is only vertical stratification $\left(d_{z 2}=\Pi\right)$. This limit case belongs to the second solution as shown in figure 4. In the upper limit of prolate vortices $(\mu=0)$ there is no displacement stratification $\left(d_{z 1}=1\right)$ and there is only relative vorticity $\left(\tilde{\zeta}_{a 1}=\Pi\right.$, $\tilde{\zeta}=\Pi-1)$.

The solutions with the negative sign in $(3.3 a)$, which may be denoted as $\tilde{\zeta}_{a 1}^{-}$, have $\Pi<0$, correspond to the so-called anomalous wind (Gustafson 1953; Fultz 1991), and are therefore $\tilde{\zeta}_{a 1}^{-}(\Pi, v)=-\tilde{\zeta}_{a 1}(-\Pi, v)$. These solutions are inertially unstable.

In region $4\left(\mathrm{~A}_{4}\right.$ and $\left.\mathrm{B}_{4}\right)$ the vortex is hyperbolic, the solution is also unique and given by $\tilde{\zeta}_{a 1}$ (figure 2 ) and $d_{z 1}$ (figure 4). In this region $\tilde{\zeta}_{a 1}<0$ and $d_{z 1}<0$, so that the flow is inertially and statically unstable. Since $\tilde{\zeta}_{a 1}(-\Pi, v)=-\tilde{\zeta}_{a 1}(\Pi, v)$ the flow is inertially stable in the region corresponding to $\mathrm{A}_{4}$ but for $\Pi<0$, however, static instability persists since $d_{z 1}(-\Pi, \mu)=d_{z 1}(\Pi, \mu)$. 


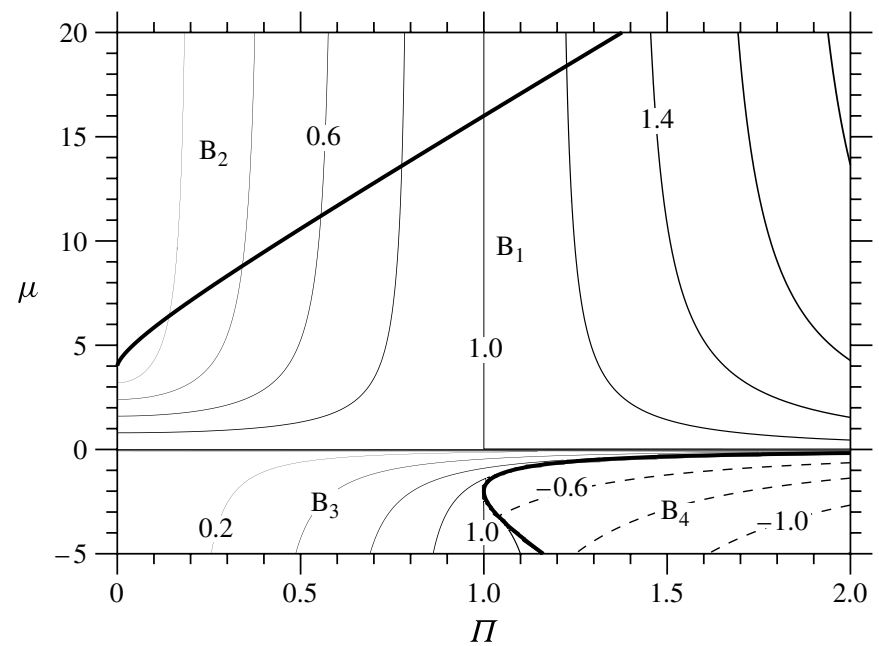

FIGURE 4. Solutions $d_{z 1}(\Pi, \mu)$ in regions $\mathrm{B}_{1}$ and $\mathrm{B}_{4}$, and $d_{z 2}(\Pi, \mu)$ in regions $\mathrm{B}_{2,3}$. The curve $\Pi_{d}(\mu)$ (continuous thick line) is included. Contour interval $\Delta=0.2$. Note that the union of functions $d_{z 1}$ and $d_{z 2}$ is continuous along the branch $\Pi_{d}(\mu>0)$ but discontinuous along $\Pi_{d}(\mu<0)$. Stratification $d_{z 2} \rightarrow \Pi$ as $\mu \rightarrow \infty$.

In regions $\mathrm{A}_{2}$ and $\mathrm{A}_{3}$ the discriminant $D_{\zeta}<0$ and there are three distinct real roots $\left\{\zeta_{a 2}, \zeta_{a 3}, \zeta_{a 4}\right\}$. To obtain these solutions we define first the angle

$$
\theta_{\zeta}(\Pi, v) \equiv \arccos \left[2 v \Pi\left(\frac{1-4 v}{3}\right)^{-3 / 2}\right] .
$$

Note that $D_{\zeta}(\Pi, v)<0$ implies that $-1<2 v \Pi[(1-4 v) / 3]^{-3 / 2}<1$ and therefore $0<\theta_{\zeta}<\pi$. The three solutions are

$$
\begin{gathered}
\tilde{\zeta}_{a 2}(\Pi, v)=2 \sqrt{\frac{1-4 v}{3}} \cos \frac{\theta_{\zeta}(\Pi, v)}{3}, \\
\tilde{\zeta}_{a 3}(\Pi, v)=-2 \sqrt{\frac{1-4 v}{3}} \cos \frac{\theta_{\zeta}(\Pi, v)+\pi}{3}, \\
\tilde{\zeta}_{a 4}(\Pi, v)=-2 \sqrt{\frac{1-4 v}{3}} \cos \frac{\theta_{\zeta}(\Pi, v)-\pi}{3} .
\end{gathered}
$$

Noticing that $\arccos (x)=\pi-\arccos (-x)$, we find the antisymmetric relations $\tilde{\zeta}_{a 2}(-\Pi, v)=-\tilde{\zeta}_{a 4}(\Pi, v)$ and $\tilde{\zeta}_{a 3}(-\Pi, v)=-\tilde{\zeta}_{a 3}(\Pi, v)$. These symmetry relations, together with $\tilde{\zeta}_{a 1}(-\Pi, v)=-\tilde{\zeta}_{a 1}(\Pi, v)$, prove the antisymmetry of the solutions $\tilde{\zeta}_{a i}$.

Consistently with the existence of three real roots $\left\{\zeta_{a 2}, \zeta_{a 3}, \zeta_{a 4}\right\}$, in regions $\mathrm{A}_{2}$ and $\mathrm{A}_{3}$ the discriminant of the stratification equation $D_{d}<0$ and there are also three distinct real roots $\left\{d_{z 2}, d_{z 3}, d_{z 4}\right\}$. To obtain these solutions we define the function

$$
\gamma(\Pi, \mu) \equiv \operatorname{sgn}(\mu-4)\left[\mu \Pi^{2}\left(\frac{6}{\mu-4}\right)^{3}-1\right]
$$

which is valid for $\mu<0$ and $\mu>4$. Let the phase function

$$
\theta_{d}(\Pi, \mu) \equiv \arccos \gamma(\Pi, \mu) .
$$




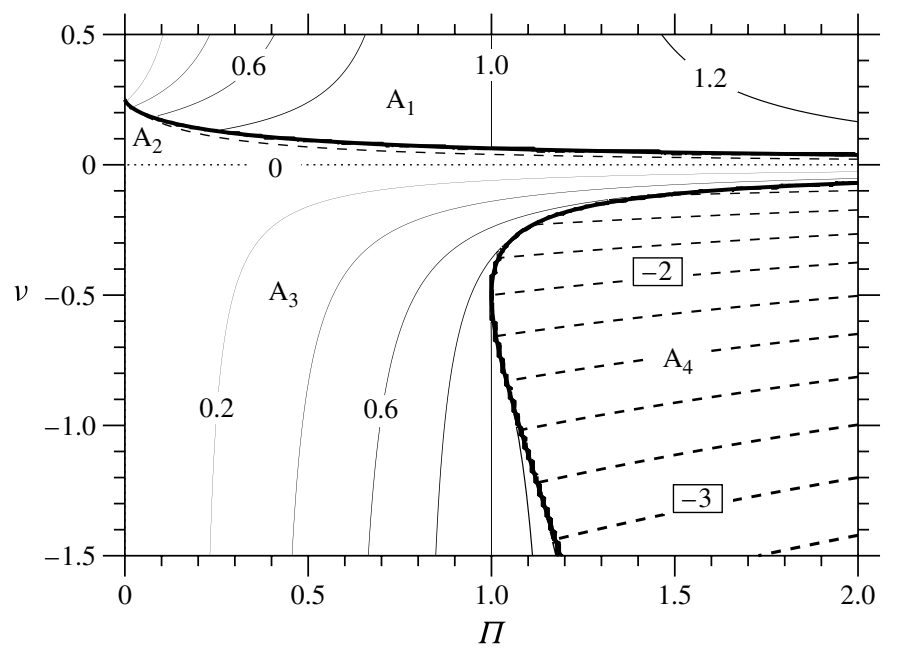

FIGURE 5. Solution $\tilde{\zeta}_{a 3}(\Pi, v)$ in regions $\mathrm{A}_{2}$ and $\mathrm{A}_{3}$. For completeness the unique solution $\tilde{\zeta}_{a 1}(\Pi, v)$ is included in regions $\mathrm{A}_{1}$ and $\mathrm{A}_{4}$ as well as the curve $\Pi_{\zeta}(v)$. Contour interval $\Delta=0.2$.

Then the solutions $\left\{d_{z 2}^{\prime}, d_{z 3}^{\prime}, d_{z 4}^{\prime}\right\}$ are

$$
\begin{gathered}
d_{z 2}^{\prime}(\Pi, \mu)=\frac{2}{3}|\mu-4| \cos \frac{\theta_{d}(\Pi, \mu)}{3}-\frac{\mu-4}{12}, \\
d_{z 3}^{\prime}(\Pi, \mu)=-\frac{2}{3}|\mu-4| \cos \frac{\theta_{d}(\Pi, \mu)+\pi}{3}-\frac{\mu-4}{12}, \\
d_{z 4}^{\prime}(\Pi, \mu)=-\frac{2}{3}|\mu-4| \cos \frac{\theta_{d}(\Pi, \mu)-\pi}{3}-\frac{\mu-4}{12} .
\end{gathered}
$$

Finally, we rearrange these solutions in such a way that the pairs of solutions $\left(\tilde{\zeta}_{a i}, d_{z i}\right)$ satisfy relation (4.5). The rearrangement is $d_{z 2} \equiv d_{z 2}^{\prime}, d_{z 3} \equiv d_{z 4}^{\prime}, d_{z 4} \equiv d_{z 3}^{\prime}$ for $\mu>0$, and $d_{z 2} \equiv d_{z 3}^{\prime}, d_{z 3} \equiv d_{z 2}^{\prime}, d_{z 4} \equiv d_{z 4}^{\prime}$ for $\mu<0$. We comment next on the solutions $\left\{\tilde{\zeta}_{a 2}, d_{z 2}\right\}$, $\left\{\tilde{\zeta}_{a 3}, d_{z 3}\right\}$, and $\left\{\tilde{\zeta}_{a 4}, d_{z 4}\right\}$, in regions 2 and 3 .

Solutions $\left\{\tilde{\zeta}_{a 2}, d_{z 2}\right\}$ in regions 2 and 3 (figures 2 and 4) correspond to vortices which are inertially stable $\left(\tilde{\zeta}_{a 2}>0\right.$, figure 2 , regions $\mathrm{A}_{2}$ and $\left.\mathrm{A}_{3}\right)$ and statically stable $\left(d_{z 2}>0\right.$, figure 4 , regions $\mathrm{B}_{2}$ and $\left.\mathrm{B}_{3}\right)$. Note that $\tilde{\zeta}_{a 2}(\Pi, v) \rightarrow 1$ and $d_{z 2}(\Pi, \mu) \rightarrow \Pi$ as $\nu \rightarrow 0$ and $\mu \rightarrow \pm \infty$, respectively. As can be seen from figures 2 and 4 the solutions $\left\{\tilde{\zeta}_{a 2}, d_{z 2}\right\}$ are the natural continuity of the unique solutions $\left\{\tilde{\zeta}_{a 1}, d_{z 1}\right\}$ in region 1 , that is, $\left\{\tilde{\zeta}_{a 2}, d_{z 2}\right\}$ and $\left\{\tilde{\zeta}_{a 1}, d_{z 1}\right\}$ are continuous along the boundary separating regions 1 and 2 , that is, along curves $\Pi_{\zeta}(v>0)$ and $\Pi_{d}(\mu>0)$. Note however that in region 2 these solutions correspond to very oblate elliptic vortices and in region 3 correspond to hyperbolic vortices.

Solutions $\left\{\tilde{\zeta}_{a 3}, d_{z 3}\right\}$ correspond to elliptic vortices in region 2 which are inertially unstable $\left(\tilde{\zeta}_{a 3}<0\right.$, figure 5, region $\left.\mathrm{A}_{2}\right)$ and statically unstable $\left(d_{z 3}<0\right.$, figure 6 , region $\mathrm{B}_{2}$ ), and to hyperbolic vortices in region 3 which are inertial and statically stable $\left(\tilde{\zeta}_{a 2}>0\right.$, figure 5 , region $\mathrm{A}_{3}$; and $d_{z 2}>0$, figure 6 , region $\left.\mathrm{B}_{3}\right)$. These solutions are discontinuous along curve $\Pi_{\zeta}(v)$ relative to solutions $\left\{\tilde{\zeta}_{a 1}, d_{z 1}\right\}$ in regions 1 and 2. 


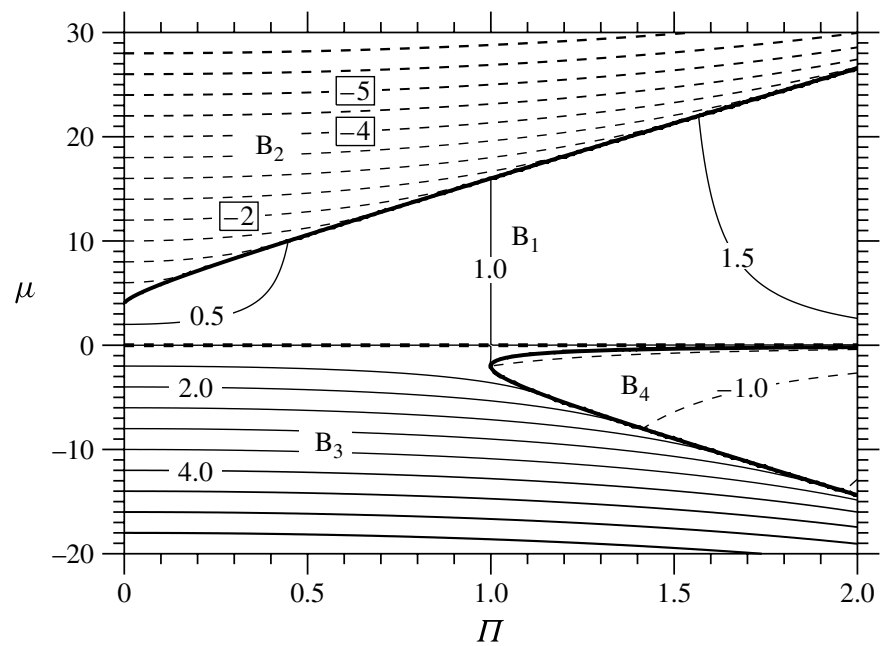

FIGURE 6. Solution $d_{z 3}(\Pi, \mu)$ in regions $\mathrm{B}_{2}$ and $\mathrm{B}_{3}$. For completeness the unique solution $d_{z 1}(\Pi, \mu)$ is included in regions $\mathrm{B}_{1}$ and $\mathrm{B}_{4}$. The curve $\Pi_{d}(\mu)$ is included. The contour interval has been increased to $\Delta=0.5$ to better represent the high values of $d_{z 3}$ in region $\mathrm{B}_{2}$.

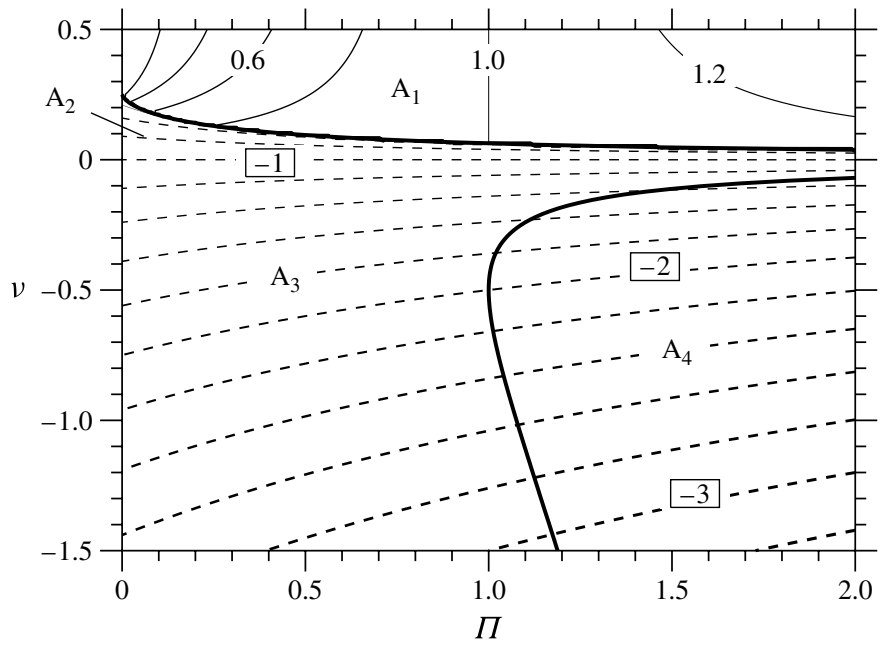

FIgURE 7. As in figure 5 but for solution $\tilde{\zeta}_{a 4}(\Pi, v)$ in regions $\mathrm{A}_{2}$ and $\mathrm{A}_{3}$. Contour interval $\Delta=0.2$.

For $\Pi<0$ vortices in region $\mathrm{A}_{2}$ are inertially stable but statically unstable. Note also that, consistently with (4.5), $d_{z 3} \rightarrow \pm \infty$ as $\tilde{\zeta}_{a 3} \rightarrow \pm 0$.

Finally, solutions $\left\{\tilde{\zeta}_{a 4}, d_{z 4}\right\}$ (figures 7 and 8) correspond to oblate elliptic vortices (region 2) and hyperbolic vortices (region 3) which are in both cases inertially and statically unstable. These solutions are the natural continuity of the unique solutions $\left\{\tilde{\zeta}_{a 1}, d_{z 1}\right\}$ in region 4 , that is, $\left\{\tilde{\zeta}_{a 4}, d_{z 4}\right\}$ and $\left\{\tilde{\zeta}_{a 1}, d_{z 1}\right\}$ are continuous along the boundary separating regions 3 and 4 , that is, along curves $\Pi_{\zeta}(v<0)$ and $\Pi_{d}(\mu<0)$. Note that $\tilde{\zeta}_{a 4}(\Pi, v=0)=-1$, so that, consistently with $(4.5), d_{z 4}(\Pi, \mu \rightarrow \pm \infty) \rightarrow-\Pi$. 


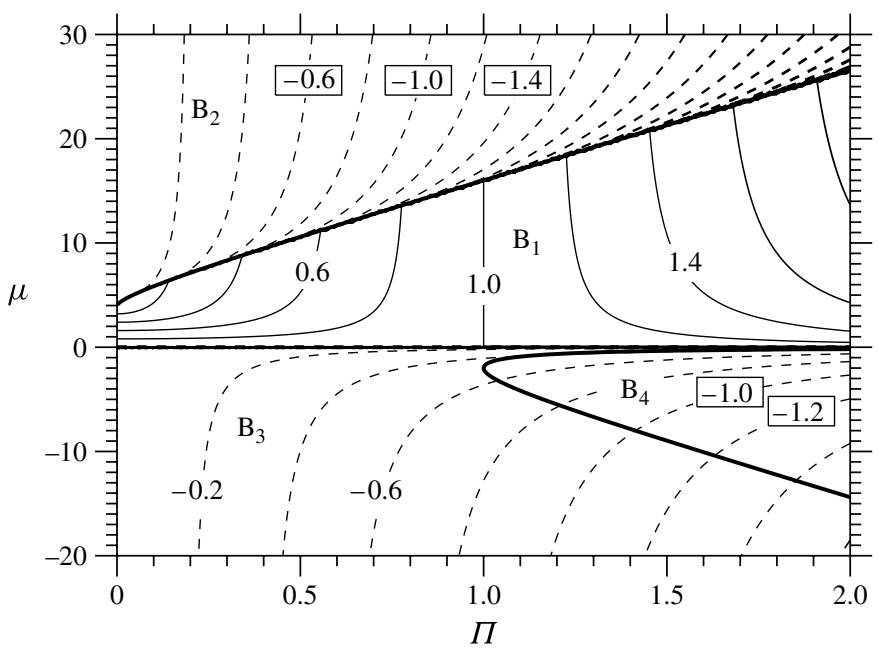

FIgURE 8. As in figure 6 but for solution $d_{z 4}(\Pi, \mu)$. Contour interval $\Delta=0.2$.

To verify these results we note that the trivial solution $\tilde{\zeta}_{a}=d_{z}=\Pi=1$, which is independent of $\mu$ and $v$, occurs for $v \geqslant 1 / 2^{4}$ in $\tilde{\zeta}_{a 1}$, for $-1 / 2 \leqslant v \leqslant 1 / 2^{4}$ in $\tilde{\zeta}_{a 2}$ (figure 2), and for $v \leqslant-1 / 2$ in $\tilde{\zeta}_{a 3}$ (figure 5); whereas it occurs for $0 \leqslant \mu \leqslant 2^{4}$ in $d_{z 1}$, for $\mu \leqslant-2$ and $\mu \geqslant 2^{4}$ in $d_{z 2}$ (figure 4), and for $-2 \leqslant \mu \leqslant 0$ in $d_{z 3}$ (figure 6). These results show that, even assuming a steady state, different elliptic pressure distributions provide an identical constant PV field. Thus, in this sense, PV inversion does not have a unique solution. The solution is unique, however, for ellipsoidal vortices if inertial and static stability is assumed, which disregards solutions $\left(\tilde{\zeta}_{a 3}, d_{z 3}\right)$ and $\left(\tilde{\zeta}_{a 4}, d_{z 4}\right)$ for positive aspect ratios.

The existence of multi-valued solutions is also useful in distinguishing balanced vortical motion (usually associated with potential vorticity anomalies) from inertia-gravity wave motion. Inertia-gravity wave motion may be defined as having zero PV anomalies (Viúdez 2012). As wave motion is excluded from the steady-state solutions found here, zero PV anomalies $(\varpi=0, \Pi=1)$ imply the state of no motion $(\tilde{\zeta}=\mathscr{D}=0)$. This is the case of solutions $\left(\tilde{\zeta}_{a 1}, d_{z 1}\right)$ and $\left(\tilde{\zeta}_{a 2}, d_{z 2}\right)$ which are all equal to 1 when $\Pi=1$. However, for very oblate elliptic vortices with aspect ratio $v<1 / 4^{2}$ $\left(\mu>4^{2}\right)$ solutions $\left(\tilde{\zeta}_{a 3}, d_{z 3}\right)$ and $\left(\tilde{\zeta}_{a 4}, d_{z 4}\right)$ differ from 1 , hence they may be associated with vortical flow despite having zero PV anomaly. These solutions are both inertially and statically unstable and therefore develop inertial and convective instabilities, time variability permitted. Thus it is more accurate to associate PV anomalies with stable balanced vortical flow, which excludes the above unstable vortical solutions. For hyperbolic vortices, which have negative ratios, there is a region in the $(\Pi, v)$ - and $(\Pi, \mu)$-spaces where three different pressure solutions are possible associated with the same constant PV distribution. However, only two of them are inertially and statically stable.

The multi-valued condition of the PV inversion problem implies also that some care must be taken when using algorithms involving PV inversion. For example, the PV initialization approach (Viúdez \& Dritschel 2003) is an algorithm for initializing geophysical balanced vortices in numerical models, which avoids the generation of spurious inertia-gravity waves. In this approach, and during the initialization period, 
the aspect ratio of the vortices (say $\mu$ ) is kept fixed whereas the PV density of every fluid particle (that is, in a Lagrangian way) is forced to increase up to its prescribed value. Thus, it is possible that, at some stage during the initialization period, the vortex enters the multi-valued region in the $(\mu, \Pi)$-space. In the PV initialization approach this situation is avoided by the PV inversion numerical algorithm which explicitly assumes balanced flow as well as inertial and static stability.

\section{Concluding remarks}

In this work we have seen that axisymmetric piecewise-constant PV geophysical vortices, in steady gradient balance, admit elliptic and hyperbolic interior solutions. The uniqueness and stability of the solutions depend on the PV and vortex aspect ratio. For every PV value there is a critical aspect ratio beyond which three different geopotential solutions are possible. Thus, a balance condition (such as the gradient balance) does not suffice to ensure the uniqueness of the solution via PV inversion. Stability conditions are also required.

Obviously, a steady ellipsoidal vortex in gradient balance cannot change its aspect ratio and, while preserving its $\mathrm{PV}$, move in a continuous way from the balanced single-valued solution region to the multi-valued solution region. However, local rates of change in the dynamical quantities may be considered by introducing, for example, plane gravity waves in the vortex domain. If the horizontal wavelength of these waves is much larger than the vortex diameter the vortex remains very approximately ellipsoidal while experiencing vertical stretching forced by the waves. If, furthermore, the vortex is originally in the unique solution region of the $\mathrm{PV}$-aspect ratio space, but with its aspect ratio very close to the critical value, an increase in the vertical extent may cause the vortex to exceed the critical value and enter the multi-valued solution region. PV inversion algorithms may experience problems under this circumstance. Care must therefore be taken when PV tools, such as the PV initialization or optimal PV balance (Viúdez \& Dritschel 2004) approaches, are used for prolate spheroid vortices beyond the critical aspect ratio. A stable vortex in the multi-valued region must however remain stable since moving to any unstable solution would imply a discontinuous jump, compatible with PV conservation, in its vertical vorticity and stratification values.

The solutions and analysis of the geopotential $\Phi$ provided here apply only to the interior part of the vortex, where the PV anomaly $\varpi \neq 0$. Solutions for the vortex exterior, where $\varpi=0$ and such that $\Phi \rightarrow 0$ as the distance to the vortex centre $r^{2}+\hat{z}^{2} \rightarrow \infty$, seem to be more difficult to find. They are however necessary in order to complete the geophysical vortex picture based on PV inversion. Approximate solutions for spherical vortices based on the quasi-geostrophic (Thorpe \& Bishop 1994), semigesotrophic (Thorpe \& Bishop 1995), and other approximations (Viúdez 2008), have already been found, but the exact exterior closed-form solutions still remain an important theoretical challenge.

\section{Acknowledgements}

I thank two anonymous referees for their very helpful comments. Partial support has come from the Spanish Ministerio de Ciencia e Innovación (grant CTM201128867). 


\section{REFERENCES}

Beltrami, E. 1871 Sui principii fondamentali dell'idrodinamica razionali. Mem. Accad. Sci. Inst. Bologna 1, 431-476.

Bishop, C. H. \& ThORPE, A. J. 1994 Potential vorticity and the electrostatics analogy: Quasi-geostrophic theory. Q. J. R. Meteorol. Soc. 120, 713-731.

CASEy, J. \& NAghdi, P. M. 1991 On the Lagrangian description of vorticity. Arch. Rat. Mech. Anal. 115, 1-14.

ERTEL, H. 1942 Ein neuer hydrodynamischer erhaltungssatz. Naturwissenschaften 30, 543-544.

FultZ, D. 1991 Quantitative nondimensional properties of the gradient wind. J. Atmos. Sci. 48, 869-875.

Gustafson, A. F. 1953 On anomalous winds in the free atmosphere. Bull. Am. Meteorol. Soc. 34, 196-201.

Holton, J. R. 2004 An Introduction to Dynamic Meteorology. Elsevier.

Hoskins, B. J., McIntyre, M. E. \& Robertson, A. W. 1985 On the use and significance of isentropic potential-vorticity maps. Q. J. R. Meteorol. Soc. 111, 877-946.

KuRganskiY, M. V. \& TATARSKAYA, M. S. 1987 The potential vorticity concept in meteorology: A review. Izv. Atmos. Ocean. Phys. 23, 587-606.

McWilliams, J. C., Weiss, J. B. \& Yavneh, I. 1999 The vortices of homogeneous geostrophic turbulence. J. Fluid Mech. 410, 1-26.

MüLLER, P. 1995 Ertel's potential vorticity theorem in physical oceanography. Rev. Geophys. 33, 67-97.

Reinaud, J. N., Dritschel, D. G. \& Koudella, C. R. 2003 The shape of vortices in quasi-geostrophic turbulence. J. Fluid Mech. 474, 175-192.

Rossby, C.-G. 1936 Dynamics of steady ocean currents in the light of experimental fluid mechanics. Pap. Phys. Oceanogr. Meteor. 5, 1-43.

Rossby, C.-G. 1940 Planetary flow patterns in the atmosphere. Q. J. R. Meteorol. Soc. 66 (suppl), $68-87$.

Santurette, P. \& Georgiev, C. G. 2005 Weather Analysis and Forecasting. Applying Satellite Water Vapor Imagery and Potential Vorticity Analysis. Elsevier Academic.

Thorpe, A. J. \& Bishop, C. H. 1994 Potential vorticity and the electrostatics analogy - quasi-geostrophic theory. Q. J. R. Meteorol. Soc. 120, 713-731.

Thorpe, A. J. \& Bishop, C. H. 1995 Potential vorticity and the electrostatics analogy - Ertel-Rossby formulation. Q. J. R. Meteorol. Soc. 121, 1477-1495.

VIÚDEZ, A. 2001 The relation between Beltrami's material vorticity and Rossby-Ertel's potential vorticity. J. Atmos. Sci. 58, 2509-2517.

VIÚDEZ, A. 2008 The piecewise constant symmetric potential vorticity vortex in geophysical flows. J. Fluid Mech. 614, 145-172.

VIÚdEZ, A. 2012 Potential vorticity and inertia-gravity waves. Geophys. Astrophys. Fluid Dyn. 106, $67-88$.

Viúdez, A. \& Dritschel, D. G. 2003 Vertical velocity in mesoscale geophysical flows. J. Fluid Mech. 483, 199-223.

ViÚDEZ, A. \& DRITSChEL, D. G. 2004 Optimal potential vorticity balance of geophysical flows. J. Fluid Mech. 521, 343-352.

WoAn, G. 2003 The Cambridge Handbook of Physics Formulas. Cambridge University Press. 